\title{
GUÍA METOdOLÓGICA DE LA GESTIÓN DE DESPERDICIOS EN UNA PYME
}

\section{METHODOLOGYCAL GUIDE OF WASTE MANAGEMENT IN A PYME}

Juhlyanis Jiménez ${ }^{1}$

Víctor Gisbert Soler ${ }^{2}$

1. Ingeniera Industria. Universidad Tecnológica de Santiago, República Dominicana. Máster en Ingeniería en Organización y Logística, Universidad Politécnica de Valencia, España.

E-mail:

juhjipe@epsa.upv.es

2. Doctor Ingeniero Industrial. Departamento de Estadística e Investigación Operativa Aplicadas y Calidad. Universidad Politécnica de Valencia, España. E-mail: vgisber@eio.upv.es

\section{Citación sugerida:}

Jiménez, J. y Gisbert Soler, V. (2017). Guía metodológica de la cuestión de desperdicios en una pyme. 3C Empresa: investigación y pensamiento crítico, Edición Especial, 57-63. DOI: <http://dx.doi.org/10.17993/3cemp.2017.especial.57-63/>. 


\section{RESUMEN}

Se conoce como desperdicio aquellas actividades que consumen recursos pero que no agregan ningún valor al producto o servicio que está generando dentro de una empresa. Existen al menos siete desperdicios a los cuales las empresas que buscan la excelencia de sus procesos se enfrentan cotidianamente. Pero ¿Cómo una empresa en pro de insertarse al mercado aprovecharía más sus recursos para ser competitivos? ¿Se optimizarían los procesos a través de la gestión de desperdicio?

\section{ABSTRACT}

It is known as waste activities that consume resources but do not add any value to the product or service that is generated within a company. There are at least nine wastes that businesses face on a daily basis. But how would a company take more advantage of its resources, managing or eliminating waste? Would it imply that waste management would be more competitive for companies? Would processes be optimized through waste management?

\section{PALABRAS CLAVE}

Desperdicio, Eliminar, Gestión, Recursos, Competitividad, Optimizar.

\section{KEY WORDS}

Waste, Remove, Management, Resources, Competitiveness, Optimize. 


\section{INTRODUCCIÓN}

Tras realizar varias investigaciones sobre el tema de gestión de desperdicios se puede notar el auge que ha tomado en las empresas por desarrollar una cultura de gestión sostenible encaminada a reducir y/o eliminar en sus procesos las actividades que consumen recursos (ya sea espacio, equipo, tiempo, etc.) pero que no agregan valor, mermando la eficiencia y productividad en su gestión. En este caso, las pequeñas y medianas empresas que buscan madurar sus procesos adaptando la gestión de desperdicio como una cultura dentro de las organizaciones, requerirá de la integración y disposición de todo el equipo de trabajo, llevando a cabo una breve guía metodológica orientada a optimizar sus recursos, haciéndolas más competitivas. Con este artículo se pretende enfatizar como gestionar esos desperdicios que no añaden valor y a la vez servirá como modelo elemental para aquellas medianas y pequeñas empresas que estás encaminada a la optimización de sus procesos.

\section{GESTIÓN DE DESPEDICIO}

El desperdicio conocido también como despilfarro o Muda por los japoneses está ligada a las actividades que se realiza durante el proceso productivo que utiliza recursos como materia prima, tiempo, equipos, materiales, personas, etc., aunque desde otra perspectiva suelen verse los desperdicios en actividades que se realizan cotidianamente. La eliminación continua y sostenible de desperdicios es el principal objetivo de Lean

Mediante la utilización de la herramienta Lean Manufacturar, sistema creado por la Toyota Company que en su implementación ha logrado beneficios significativos dentro de la empresa y tiene como principal objetivo la eliminación de desperdicio, más allá de esto, buscamos una forma de gestionar., además de que la continua eliminación de los mismo es su principal objetivo para mejorar los procesos.

En este sentido, para hacer una buena gestión debemos tener claro algo ¿Cuáles son los desperdicios que se pueden gestionar y cómo?

Tener una buena gestión de desperdicio en las organizaciones permite tener mayor fluidez de las operaciones que se realizan, además que contribuye a que estas sean más eficientes.

Aunque suele parecer a simple vista que en la empresa no existe desperdicio alguno, es sensato reconocer que muchas de ellas no son conscientes del grado de desperdicio a los que están expuestos sus procesos productivos, así como en la gestión administrativa que se encarga por velar que los recursos puedan satisfacer la demanda interna de la empresa.

¿Pero Cómo detectar los desperdicios y cómo gestionarlos de forma eficiente?

A continuación, se muestra una guía básica y de fácil implementación para poder lograr el objetivo Lean en su empresa: 


\section{Sobre producción:}

Para evitar este tipo de desperdicio que normalmente ocurre cuando hay una mala previsión es necesario adoptar un modelo Justo a tiempo para tener una correcta planificación de los recursos y producir cuando se necesite, en las cantidades correctas.

Llevar un registro de las cantidades que se producidas ya sea por tipo de cliente para promediar las cantidades a producir para el tipo de cliente y a la vez hacer el proceso más eficaz.

\section{Esperas}

Las esperas durante el proceso productivo son otro de los factores que provocan desperdicios en la empresa cuando hay actividades que no generan valor alguno al producto. Debe llevarse a cabo un programa donde sea necesario el cumplimiento de actividades en el tiempo que se indique. Evitar el retraso de los materiales que se necesitan, de las aprobaciones administrativas e imputabilidad de los operadores. Así como establecer buenas relaciones con los proveedores son alternativas que ayudaran a no permitir que se generen cuellos de botellas en el proceso.

El tiempo es un componente que puede decidir sobre la ventaja competitividad de las empresas.

\section{Transporte}

Se debe sacar el máximo provecho a los movimientos que se realizan en la empresa, y dejar de un lado mover material de un lugar a otro sin sentido alguno. Realizar un recorrido eficiente en la zona es necesario tener una buena distribución de planta, descartar grandes almacenes y reducir el tiempo de suministro.

\section{Procesos inapropiados o sobre procesos}

En este proceso hay que tomar en cuenta la formación de los operarios en mejora continua. Esto permite que pueda detectar que tareas del proceso pueden simplificarse. El adiestramiento del operario será de gran utilidad para crear un hábito reactivo y proactivo para localizar aquellas tareas que son innecesaria en el proceso a su cargo.

\section{Exceso de Inventario}

Si queremos tener un proceso eficiente y eficaz es imprescindible optimizar los recursos. Producir de más viene dado por una mala planificación lo que genera costes elevados y poca rentabilidad.

\section{Movimientos innecesarios}

Cualquier movimiento que el operario realice aparte de generar valor agregado al producto o servicio. Incluye a personas en la empresa subiendo y bajando por documentos, buscando, escogiendo, agachándose, etc. Incluso caminar innecesariamente es un desperdicio. 


\section{Defectos}

Esto se origina por falta de la ausencia de calidad en los productos o procesos, así como la repetición o corrección de procesos, el re-trabajo en productos no conformes o devueltos por el cliente

Para eliminar este desperdicio es necesario que las áreas involucradas tengan una calidad autónoma, y sean capaces de comprometerse a inspeccionar sus procesos para prevenir una mala calidad de los mismos.

Además de los siete desperdicios fundamentales mencionados existen dos desperdicios a los cuales hay que prestarle especial atención:

7 + 1 Desaprovechamiento Este es el octavo desperdicio y se refiere a no utilizar la creatividad e inteligencia de la fuerza de trabajo para eliminar desperdicios. Cuando los empleados no se han capacitado en los 7 desperdicios se pierde su aporte en ideas, oportunidades de mejoramiento.

Es importante escuchar las ideas de los trabajadores, ya que son los que están directamente trabajando en el proceso. Capacitar y fomentar la polivalente que no es más que la capacidad que tiene un empleado de desarrollar diferentes funciones en distintos puestos de trabajo, y como consecuencia seria soporte de áreas donde se requieran sobre todo cuando se producen ausentismos.

\section{CONCLUSIONES}

En resumidas cuentas, la implementación de la gestión de los desperdicios es un tema fundamental para cualquier empresa que busca optimizar sus procesos. Siendo una herramienta que garantiza resultados exitosos haciendo de la gestión la excelencia a través del uso más eficiente y eficaz de los recursos y materiales. De esta manera se logra el aprovechamiento máximo de los mismos lo que se traduce o deriva una mayor productividad, menores costos operativos, mayor calidad en los productos y servicios entregados al mercado, más satisfacción del cliente, mayores ventas y utilidades, que sumados, incrementan los niveles de competitividad.

La implantación de modelos de gestión de este tipo se hace cada vez más urgente entre las organizaciones, y en el mundo en general, a fin de reducir los altos riesgos que por el mal aprovechamiento de los recursos estamos enfrentando y deberemos afrontar en el futuro. 


\section{ANEXOS}

Cero Desperdicios.

\section{Lean}

Manufacturing
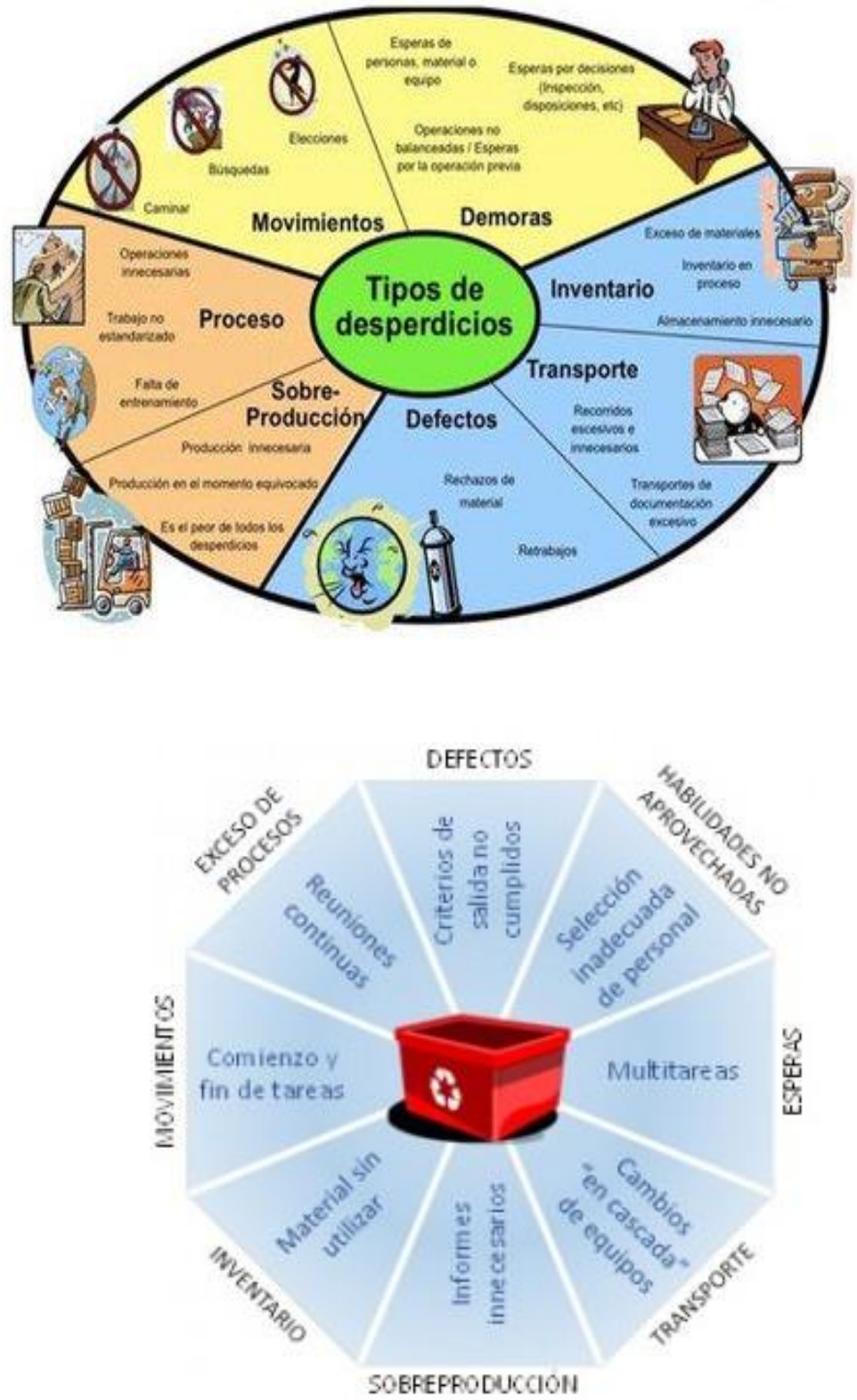


\section{REFERENCIAS BIBLIOGRÁFICAS}

1) Las claves del éxito de Toyota, Jeffrey K. Liker (Author)

2)

https://www.gestiopolis.com/gestion-de-desperdicios-y-cultura-de-maximoaprovechamiento-para-mexico/

3) http://lean-esp.blogspot.com.es/2008/09/71-tipos-de-desperdicios.html 